\title{
The CA model of the Impact on Surrounding Traffic Capacity by Residential area Opening
}

\author{
Fei Yuan ${ }^{\mathrm{a}}$, Weiwei Zheng ${ }^{\mathrm{b}}$, Yunfeng $\mathrm{Ma}^{\mathrm{c}}$, Pingji Deng ${ }^{\mathrm{d}}$ and Chunjing Liu${ }^{\mathrm{e}^{*}}$ \\ School of Hubei University of Medicine, Shiyan 442000, China. \\ a527345109@qq.com, ${ }^{b} 1142741610 @ q q . c o m,{ }^{c} 2781356051 @ q q . c o m$, \\ d1049549106@qq.com, ${ }^{\mathrm{e}}$ Icj1368@126.com \\ *The corresponding author
}

\begin{abstract}
In the case of the increasingly serious urban traffic problems, our state had issued a relevant policy on the opening of the district recently. Firstly, this paper established a suitable evaluation index system and vehicle traffic CA model; and then through the use of MATLAB programming, we finally proved that the district opening can ease the pressure on the surrounding road traffic to a certain degree.
\end{abstract}

Keywords: Road access; Community opening; Analytic hierarchy process; Cellular automata model

\section{小区开放对周边道路通行能力影响的 $\mathrm{CA}$ 模型}

袁飞 ${ }^{2}$ ，郑韦韦 ${ }^{2}$ ，马云风 ${ }^{2}$, 邓平基 ${ }^{1}$, 刘春景 ${ }^{1 *}$

1 湖北医药学院公共卫生与管理学院, 湖北十堰, 442000; 2 湖北医药学院药学院, 湖北十堰, 442000

摘要: 在现代城市交通问题日益严重的情况下，国家发布了有关小区开放的相关政策，本文首先通过建立合适的评价指 标体系和车辆通行 CA 模型等, 然后通过 MATLAB 编程求解, 研究得到小区开放在一定程度上缓解了周边道路通行的压力。

关键词: 道路通行; 小区开放; 层次分析法; 元胞自动机模型

中图分类号: 029 文献标志码: A

引言

2016 年 2 月 21 日, 国务院发布《关于进一步加强城市规划建设管理工作的若干意见》 ${ }^{[1]}$, 其中第十 六条关于推广街区制, 原则上不再建设封闭住宅小区, 已建成的住宅小区和单位大院要逐步开放等意见, 引起了广泛的关注和讨论。

除了开放小区可能引发的安保等问题外, 议论的焦点之一是: 开放小区能否达到优化路网结构, 提高 道路通行能力, 改善交通状况的目的, 以及改善效果如何。一种观点认为封闭式小区破坏了城市路网结构, 堵塞了城市 “毛细血管”, 容易造成交通阻塞。小区开放后, 路网密度提高, 道路面积增加, 通行能力自 然会有提升。也有人认为这与小区面积、位置、外部及内部道路状况等诸多因素有关, 不能一概而论。还 有人认为小区开放后, 虽然可通行道路增多了, 相应地, 小区周边主路上进出小区的交叉路口的车辆也会 增多, 也可能会影响主路的通行速度。

据此为了研究小区开放对周边道路通行的影响, 为科学决策提供定量依据, 本文建立了层次分析模型 以及元胞自动机模型 (简称 CA 模型) ${ }^{[2]}$, 通过 MATLAB 编程求解, 从不同角度定量的评价了小区开放对周 边道路通行的影响。并根据研究结果, 对小区开放进行了可行性分析, 并给出了合理化建议。 


\section{1 小区开放对周边道路通行影响的评价指标体系的建立}

首先我们模拟最理想的三岔路口模型，推导出路段流量和路段行驶时间的关系，得出小区开放对周边 道路车流量确实存在着影响, 进而我们又运用层次分析模型, 得出层次分析图, 确定各影响因素的权重, 进行一致性矩阵检验, 由于 $\mathrm{CR}=\mathrm{CI} / \mathrm{RI}<0.1$, 不一致程度在容许范围内, 所以本文所选取的车流量是对道路 通行能力有一定影响的。

\section{1 理想模型的建立与求解}

在城市道路的路段通行能力承受范围下, 我们将未开放小区时的主干道的流量设为 $Q$, 当小区开放后, 我们建立了最简单的三岔路模型, 如图 1:

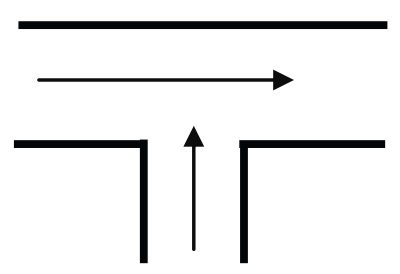

图 1 最简单的三岔路图

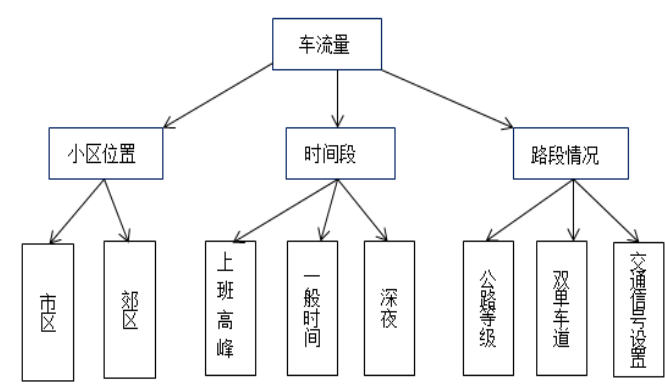

图 2 影响道路通行的三级指标

由交通流理论 ${ }^{[3]}$, 速度一密度的线性, 可知路段车流量 $(\mathrm{Q})$ 和路段车流密度 $(\mathrm{K})$ 之间的关系为 $Q=K V$, 其中 $V$ 为行车速度, $V_{f}$ 为自由流行驶的行车速度, $K_{\text {max }}$ 为路段车流密度的最大值, 则有 $V(K)=V_{f}-\frac{V_{f}}{K_{\max }} K$, 于是有 $Q(K)=V_{f} K-\frac{V_{f}}{K_{\max }} K^{2}$, 再令 $\frac{d Q}{d K}=0$, 令最大值路段的通行能力 C, 则有 $C=\frac{1}{4} V_{f} K_{\text {max }}$, 假定某路段 $\mathrm{a}$ 的长度为 $\mathrm{L}, t_{0}$ 为在自由流状态下的路段 $\mathrm{a}$ 的行驶时间, $Q=-\frac{K_{\max }}{V_{f}}\left(\frac{l}{t}\right)+K_{\max } \frac{l}{t}$, 解之得 $\frac{t_{0}}{t}=\frac{1}{2} \pm \frac{1}{2} \sqrt{1-\frac{Q}{C}}$, 于是得到路段流量与行驶所需时间的表达关系式 $\frac{Q}{C}=1-\left(\frac{2 t_{0}}{t}-1\right)^{2}$, 由此说明开放小区后由于忿路口的增加, 会增加车辆路段行驶时间, 那么路段流量 也会得到减缓, 即说明小区开放对路段的车流量是有影响的。

\section{2 层次分析模型}

我们通过建立层次分析模型 ${ }^{[4]}$ 用以评价小区开放对周边道路通行的影响。影响道路通行的因素有多个 方面, 包括周围的繁华程度, 道路所处的位置等, 层次分析模型把不同的影响因素概括为三级指标, 其中 车流量作为一级指标, 小区位置, 时间段, 路段情况作为二级指标, 小区所处位置, 时间段, 交通信号等 作为三级指标, 如上图 2:

就各级指标两两比较的得到的各级指标矩阵, 利用 MATLAB 对我们所得矩阵进行数据计算, 求得计算 结果如下表 1: 


\begin{tabular}{ccccc}
\hline $\mathbf{K}$ & $\mathbf{1}$ & $\mathbf{2}$ & $\mathbf{3}$ & $\mathbf{4}$ \\
\hline & 0.5071 & 0.9487 & 0.8452 & 0.5071 \\
$W_{K}$ & 0.8452 & 0.3162 & 0.5071 & 0.8452 \\
& 0.1690 & & 0.1690 & 0.1609 \\
\hline$\lambda_{K}$ & 3 & 2 & 3 & 3 \\
\hline $\boldsymbol{C} \boldsymbol{I}_{\boldsymbol{K}}$ & $\mathbf{0}$ & $\mathbf{0}$ & $\mathbf{0}$ & $\mathbf{0}$ \\
\hline
\end{tabular}

表 1 层次分析模型一

计算得到一致性比率 $C R=\frac{C I}{R I}<0.1$, 所以就本文所选取的各个指标体系来说, 小区开放对周边道 路有一定的影响, 此外我们还可以建立道路网络化模型, 通过比较从出发地到目的地的时间, 来说明开放 小区对人们出行时间的影响。

\section{2 小区周边道路车辆通行的 CA 模型}

20 世纪 50 年代, JohnvonNeuman 和 StanislamM. Ulam 提出元胞自动机后, 受到学术界重视。1992 年, 德国物理学家 Kai Nage1 和 Michae1 Schreckenberg 在 184 号规则基础上提出一维交通流 Nage1 Schreckenberg（NS）模型, NS 是最常用的交通流模型之一 ${ }^{[5]}$, 双向单行道 NS 模型也是一个概率元胞自动 机，每辆车的状态都由它的位置和速度所表示。

通过元胞自动机 NS 模型建立车辆通行的 CA 数学模型, 以小区外的双向单行道为研究对象, 选取合适 参数, 比较小区外的平均流量和速度的变化, 小区开放以后, 周边道路的平均车流量将会减少, 说明小区 开放对周边道路的车辆通行压力进行了一定缓解和分担, 同时周边道路的平均速度也会降低, 说明小区的 开放限制了周边道路车辆的速度。

\section{1 模型的建立}

用计算机无法直接模拟一条无限长的车道，本文没有特殊考虑，利用周期性边界构造一条环路来模拟 无限长车道的交通情况。相对开放边界条件, 周期性边界条件 (封闭环行车道) 更为简单, 更容易控制和 固定车流密度，平均速度和平均流量也不会有太大波动，便于测试不同密度下的各种参数 ${ }^{[6]}$ 。

NS模型是一个最重要的单车道CA模型。Nagel在确定性模型的基础上加入随机项. 车辆 $n$ 的位置 $x_{n}$, 速 度为 $v_{n}$, 速度 $v_{n} \in\left\{0,1,2, \cdots, v_{\text {max }}\right\}$ 为整数, 车辆 $n+1$ 在车辆 $n$ 前方, 辆车间距 $d_{n}=x_{n+1}-x_{n}$, 单元格长 7.5 米, 时间步长1秒。状态更新规则由连续的四步构成 ${ }^{[6]}$ :

(1) 加速: 如果 $v_{n}<v_{\text {max }}$ 则速度加 1 . 但不超过 $v_{\text {max }}$ 即 $v_{n}: \min \left\{v_{n}+1, v_{\text {max }}\right\}$

（2）减速（因其它车辆）：如果 $d_{n} \leq v_{n}$, 则车辆 $n$ 减速到 $d_{n}-1$, 即 $v_{n}: \min \left\{v_{n}, d_{n}-1\right\}$

(3) 随机化减速: 如果车辆 $n$ 速度大于 0 . 则以概率 $\mathrm{p}$ 减 1 , 否则不变. 即 $v_{n}:=\max \left\{v_{n}-1,0\right\}$ 以概率 $\mathrm{p}$

(4) 移动: 车辆以新速度向前移动 $v_{n}:=x_{n}+v_{n}$

第一步反映了驾驶员追求速度的一般趋势, 第二步反映了避免碰撞的意图, 第三步的随机化包含了驾 驶员的不同行为模式, 车辆以概率p减速（有速度波动、刹车或跟驰时的过度反应、加速时的随机性三种原 因）。第四步更新车辆位置, 这是能够反应真实交通现象的最小化规则集。缺少任何一条规则或改变执行 顺序就不能产生真实行为。基本图包括自由流和拥挤流两个分支，但是不能再现亚稳态，也不能再现同步 
交通相 ${ }^{[7]}$

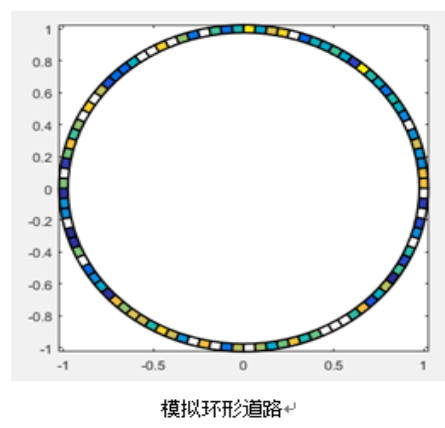

\section{2 模型的求解}

当小区不开放时, 通过 NS 模型对小区外的单车道进行模拟, 通过选取合适的环形车道上的车辆密度, 随机减速概率, 环形车道长度和模拟时间步数, 通过计算可求出环道上的平均流量和速度。

选取输入参数: 密度 $\mathrm{d} 1=0.5$, 随机减速概率 $\mathrm{p}=0.15$, 环形车道长度 $\mathrm{L}=100$, 模拟时间步数 times $=100$; 通过 MATLAB 程序计算得出环道上的平均流量 $\mathrm{Q}$ 和流速 $\mathrm{Vp}$ 分别是 0.39 和 0.78 .

当小区开放的时候, 可以抽象的看做是, 在小区外原本单车道的基础上修建了一个交叉路口，从而形 成一段 $\mathrm{T}$ 型路面, 由于研究的是小区开放对周边道路的影响, 所以依旧以小区外的单车道为研究对象, 再 次进行小区开放后的 NS 模型的模拟, 输入合适的参数, 计算出小区开放后环道上的平均流量和平均流速, 由于另一个路口的加入, 所以环形车道上的车辆密度增加, 我们选取该车辆密度 $\mathrm{d} 2=0.55$, 其他参数保持不 变, 计算结果平均流量 Q2 和平均流速 vp2 分别是 0.34 和 0.61 . 调用函数: $\mathrm{d}=0.55 ; \mathrm{p}=0.15 ; \mathrm{L}=100 ;$ times $=100 ;$ edit $\mathrm{NS}$;

通过对比 Q1 和 Q2 的值, vp1 和 vp2 的值, 说明小区的开放, 分担了周边道路的车辆压力, 同时小区 的开放也限制了周边道路的车辆速度。

\section{3 不同类型小区开放前后对道路通行的影响}

根据小区内部道路结构, 假拟了三种不同的小区, 应用层次分析法, 分别得到组合权向量, 依次为 0.9201, 0.5338, 0.6323, 由此选出通行最佳小区, 再运用第二问的元胞自动机 NS 模型, 选取合适的参数, 比较不同类型开放小区对周边道路的影响, 类型一小区对周边道路的车辆通行压力的分担能力最大, 同时 对周边道路车辆的速度限制也最大。

根据小区内部道路结构, 假拟了三种不同的小区, 如下图 3:

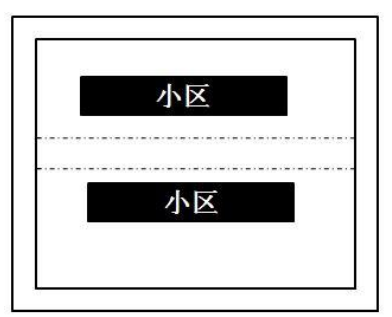

小区开放理想模型一

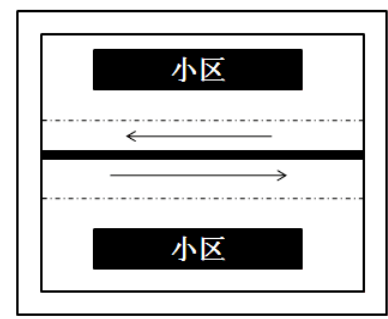

小区开放理想模型二

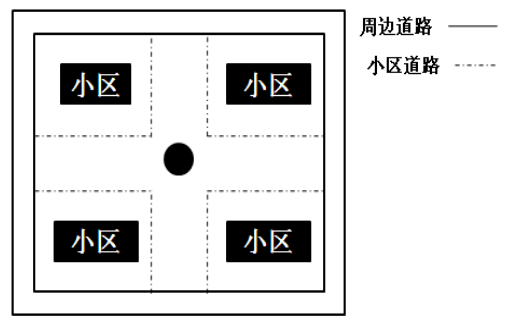

小区开放理想模型三

图 3 小区开放理想模型

那么把这三种不同小区作为层次分析法中的方案层, 把岔路口的时间延误, 车流量, 交通信号的设置 作为准则层, 把选择哪一种小区的建设当做目标层。层次结构图如下图 3: 由结构图得到各级指标之间的 
比较矩阵, 由比较阵计算出权向量 $W_{k}$, 最大特征根 $\lambda_{k}$ 和一致性指标 $C I_{k}$, 结果如下表 2 :

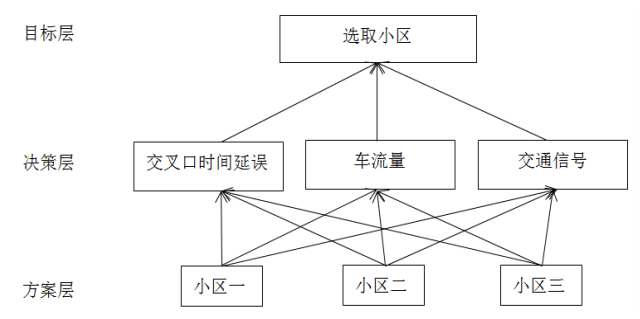

图 4 不通小区层次结构图

\begin{tabular}{lccc}
\hline $\mathrm{K}$ & 1 & 2 & 3 \\
\hline \multirow{2}{*}{$W_{K}$} & 0.1228 & 0.2181 & 0.9682 \\
& 0.8650 & 0.3778 & 0.3301 \\
& 0.3333 & 0.6667 & 0.6667 \\
\hline$\lambda_{K}$ & 3.0142 & 3.0183 & 3 \\
\hline $\boldsymbol{C I}_{\boldsymbol{K}}$ & 0.0071 & 0.00915 & 0 \\
\hline
\end{tabular}

表 2 层次分析模型二

计算可得 $C I_{k}$ 均可通过一致性检验, 对于小区一的权重用权向量表示为 $W^{(1)}$, 所以方案在目标中的组 合的权重应为他们相应项两两乘机之和, 即 $0.1288 \times 0.1925+0.865 \times 0.9623+0.3333 \times 0.1925=0.9201$; 同样的计算出, 小区二, 小区三在目标中组合权重分别为 $0.5338,0.6323$, 表明在小区的选择中小区一占 的权重近于 1 , 应选择小区一。

通过层次分析法, 得到了最佳小区, 再结合元胞自动机 NS 模型, 给予不同类型小区不同的车辆密度 值, 并通过问题二已建立的 NS 模型进行计算, 每种类型的开放小区, 依旧看做是在小区外原本双向单行 道的基础上修建了一个交叉路口, 从而形成一段 $\mathrm{T}$ 型路面, 由于研究的是小区开放对周边道路的影响, 所 以依旧以小区外的双向单行道为研究对象, 输入合适的参数, 计算出三种类型小区开放后环道上的平均流 量和平均流速, 模拟环形车道的计算结果如下表 3:

\begin{tabular}{|c|c|c|c|c|c|c|}
\hline & $d$ & $p$ & $\mathrm{~L}$ & times & $Q$ & $\mathrm{~V} R$ \\
\hline 小区- & 0.7 & 0.15 & 100 & 100 & 0.2420 & 0.3457 \\
\hline 小区二 & 0.6 & 0.15 & 100 & 100 & 0.3206 & 0.5343 \\
\hline 小区三 & 0.55 & 0.15 & 100 & 100 & 0.3390 & 0.6054 \\
\hline
\end{tabular}

表 3 层次分析模型三

根据上述模拟结果可以知道, 由于小区所占权重越大，我们赋予的环形车道密度值越大，开放小区所 占权重越大对环形车道的平均车流量和速度影响也大, 小区一的影响最大, 相对应的环形车道平均车流量 和速度最小, 说明小区一对小区外车辆通行压力的分担缓解的能力最大, 最有利于缓解交通堵塞, 相应地, 由于该小区的开放，小区周围路段的平均车速也会适当降低。

我们得出小区的类型对于小区开放产生的效果是有影响的。小区的开放能不能改善城市的交通，与小 区面积, 小区的地理位置繁华度, 及小区的内部道路结构宽广度等因素有着密切的联系。另外对于城市中 旧城、旧区、旧建筑的改造和城市的新的建筑建设更要有前瞻性, 据可行性分析, 结合问题三的模型和不 同社会人士对小区开放的自身利益化方面的考虑, 结果表明, 大家对现在居住着的城市小区都不太愿意开 放，所以，小区的开放可以放在新地产的开发上。 


\section{致谢:}

本文由湖北医药学院研究生启动基金资助，基金号（）

\section{Acknowledgement:}

This work was supported by the initial project for postgraduates of Hubei University of Medicine (NO. 2014QDJZR17).

\section{参考文献:}

[1]程思炜, 吴赋. 中国不再建设封闭小区已建小区要逐步开放 [J]. 南方网, 2016.

[2]贾斌, 高自由. 基于元胞自动机的交通系统建模与模拟 [M]. 科学出版社. 2007. 01.

[3] 马俊. 交通流理论基础 [M]. 中国人民公安大学出版社. 2004.01.

[4]姜启源, 谢金星, 叶俊. 数学模型 [M]. 北京: 高等教育出版社, 2011 .

[5]叶冬, 樊镭. 一维单车道交通流元胞自动机模型综述 [J]. 电子与控制, 2013, 05:23-29.

[6]Nage1 K. Schreckenberg M .A cellular automaton model for freeway traffic [J]. Journal of Physics I France, 1992, 2:2221-2229.

[7]张发, 宣慧玉. 基于元胞自动机的交通模型综述 [J].系统工程, 2004, 22 (12) :77-84.

[8]司守奎, 孙兆亮. 数学建模算法与应用 $[M]$. 北京: 国防工业出版社, 2015.4.

\section{References:}

[1] Cheng Siwei, Wu Fu. China is no longer building a closed area, that has been built to gradually open community [J]. Southern Network, 2016.

[2] Jia Bin, high freedom. Modeling and simulation of traffic system based on cellular automata [M]. Science Press .2007.01.

[3] Ma Jun. Traffic flow theory foundation [M]. Chinese People's Public Security University Press .2004.01.

[4] Jiang Qiyuan, Xie Jinxing, Ye Jun. Mathematical model [M]. Beijing: Higher Education Press, 2011.

[5] Ye Dong, Fan Lei. Overview of one-dimensional single-lane traffic flow cell automata model [J]. Electronics \& Control, 2013, 05: 23-29.

[6] Nagel K. Schreckenberg M.A cellular automaton model for freeway traffic [J]. Journal of Physics I France, 1992, 2: 2221-2229.

[7] Zhang Fa, Xuan Huiyu. Traffic Model Based on Cellular Automata [J]. Systems Engineering, 2004, 22 (12): 77-84.

[8] Shi Shoukui, Sun Zhaoliang. Mathematical modeling algorithm and application [M]. Beijing: National Defense Industry Press, 2015.4. 